\title{
Potential of dietary supplementation with berries to enhance immunity in humans: A mini-review
}

\author{
Athena Donga ${ }^{\mathrm{a}}$, Jianhua $\mathrm{Yu}^{\mathrm{b}}$, Xiao Chen ${ }^{\mathrm{a}}$ and Li-Shu Wang ${ }^{\mathrm{a}^{*}}$

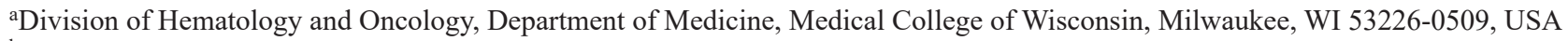 \\ ${ }^{b}$ Department of Hematology \& Hematopoietic Cell Transplantation, City of Hope National Medical Center and Beckman Research Insti- \\ tute, Duarte, CA 91010, USA \\ *Corresponding author: Li-Shu Wang, Division of Hematology and Oncology, Department of Medicine, Medical College of Wisconsin, \\ Rm C4930, 8701 Watertown Plank Rd, Milwaukee, WI, 53226, USA. E-mail: liswang@mcw.edu \\ DOI: $10.31665 /$ JFB.2021.16289
}

Received: December 19, 2021; Revised received \& accepted: December 19, 2021

Citation: Dong, A., Yu, J., Chen, X., and Wang, L.-S. (2021). Potential of dietary supplementation with berries to enhance immunity in humans: A mini-review. J. Food Bioact. 16: 19-24.

\begin{abstract}
One of the most prominent concerns that has arisen from global events such as the Coronavirus Disease 2019 (COVID-19) pandemic is how the immune system might be enhanced to provide greater protection against malignant conditions and diseases. Dietary modification is one of the major fields of research, with special focus on how diet interacts with immunity through impacts on factors such as the gut microbiome, inflammation, and nutritional imbalance. This review focuses on current research regarding the use of bioactives derived from berries as a dietary supplement to improve immunity, with discussions of relevant clinical studies. Major bioactive, metabolic compounds of focus-flavonoids, anthocyanins, alkaloids, dietary fiber, and stilbenes have demonstrated biochemical merits in modulating immunity. In addition, blueberries, goji berries, black raspberries, and cranberries, which have been extensively researched and recently gained interest for their effects on the immune system in animal and cell models, may also hold promise in providing similar benefits to humans, though the precise immunological effects have yet to be clearly determined. Overall, the field of berry research as it relates to diet and immunity shows potential, but more clinical studies will be necessary for a full understanding of the mechanisms of berry immunomodulation.
\end{abstract}

Keywords: Berries; Immune system; Diet; Inflammation; Gut microbiome.

\section{Introduction}

The international spread of Coronavirus Disease 2019 (COVID-19) has brought to the forefront concerns regarding optimal immune protection against not only COVID and similar viruses, but also other debilitating conditions which commonly afflict patients. Those with preexisting conditions that affect efficacy of the immune system, such as type II diabetes mellitus, are especially susceptible to worsened outcomes in association with their conditions after infection with viruses such as SARS-CoV-2 (Yazdanpanah et al., 2020). Additionally, tentative studies show that patients with autoimmune disease may be at increased risk for severe COV-
ID-19 or mortality due to COVID-19 (Liu et al., 2020a). Although great effort has been dedicated to the development of vaccines, with much success, many human diseases still lack such effective immune protection. One method of enhancing immunity that has been under increasing research in the past decades is the potential of dietary modification or supplementation.

Recent studies regarding the interactions between diet and immunity have especially focused on the Western diet, which has also become prevalent in developed and developing countries as the accessibility and processing of food have increased. While a Western diet may have a greater range of nutrients, these nutrients are often consumed in excess and thus cause detriment to the immune system. For instance, the activation of adipocytes by weight gain 
promotes the release of proinflammatory cytokines, such as tumor necrosis factor (TNF) and interleukins (ILs); when prolonged weight gain leads to constitutive adipocyte activation, the immune system becomes downregulated such as not to constantly mount a full inflammatory response (Myles, 2014). This increases susceptibility to disease, and the immune system switches to instead a systemic low level of activation (Wypych et al., 2017). In addition to increasing disease vulnerability, this increased inflammation may promote the pathogenesis of conditions such as type 2 diabetes and inflammatory bowel disease (Ricordi et al., 2015). Another major switch that occurs with diet is in the gut microbiome, where many bacterial species reside and interact with the human immune system-one of the major functions of the gut microbiome is to protect against infection by pathogenic bacteria, and the distribution of species in the microbiome can determine whether the bacterial profile is relatively pro- or anti-inflammatory (Brown et al., 2012). Studies in both humans and animal models have shown that a highfat diet (HFD) with increased proportion of animal products promotes a more inflammatory gut microbiome profile as opposed to a diet that is low-fat and plant-based (Tilg and Moschen, 2015). Other studies corroborate this conclusion; for example, it has been demonstrated that bioactive compounds in cruciferous vegetables benefit intestinal immune function by providing ligands for the aryl hydrocarbon $(\mathrm{AhR})$ receceptor, which modulates the T-cell response against environmental insults (Hooper, 2011).

Such discussion thus leaves the question of what modifications can be made to the human diet in order to bolster the immune system and hypothetically prevent the development of detrimental conditions. A low-fat, plant-based diet as aforementioned has been extensively demonstrated to promote a healthier gut microbiome and decrease proclivity for systemic inflammation, but the precise execution of such a diet has not always been specified (Tilg and Moschen, 2015; Hooper, 2011). This review will focus on the use of berries as a dietary supplement, providing an overview of potential immunological health benefits that have been explored in human clinical trials as well as mechanisms that have been elucidated to better the understanding of how berries affect the immune system.

\section{Materials and methods}

A literature review was conducted within the Medical College of Wisconsin Libraries PubMed LinkOut database to identify articles that fulfilled all three of the following criteria: (1) clinical research performed on human subjects (in vivo), (2) treatment involved consumption of berries or bioactive compounds derived from berries, (3) outcomes assessed factors specifically related to the human immune system. Extraneous articles that did not fall under these categories were only included where they provided helpful and relevant context for human clinical studies. To ensure that current and recent research was presented in this mini-review, only articles from 2010 forward were included, with greater preference given to articles published within 2015-2021 in order to improve contemporary relevance. Authors were required to be free from significant conflicts of interest. While specific berries were not included as criteria in the initial literature search, a follow-up search with this criterion was performed when it was found that certain berries tended to appear more frequently in the relevant literature, in order to better examine the various range of effects promoted by these berries. Specific berry types and bioactive/metabolic compounds were only selected to be discussed in the article if they had a sizeable body of clinical trial-based research, which was in- tended as a limitation to the scope of the review; thus, this review may not have included certain berries if they did not appear in a significant number of relevant clinical studies. It is acknowledged that this omission could prevent the review from presenting the full body of clinical trials involving berries.

\section{Clinical studies}

\subsection{Bioactive and Metabolic Compounds}

Berries contain a number of bioactive compounds that may have effects on immune modulation (Gupta and Prakash, 2014). In addition to the more common vitamins and minerals, there are some phytochemicals that are more unique to berries and/or have been more extensively studied in berry-related contexts. One such component is flavonoids, which are metabolic products of berries that have antioxidant properties by virtue of a polyphenolic structure (Pérez-Cano and Castell, 2016). It has been tentatively shown that flavonoids can exert anti-inflammatory effects, for example by supporting normal function of the intestinal gut microbiota barrier and inhibiting cyclooxygenase $(\mathrm{COX}-2)$, the latter of which promotes inflammation via prostaglandin production (Vezza et al., 2016). Specifically, the flavonoid quercetin, which is found in berries, has been shown in clinical trials to reduce upper respiratory tract infection (URTI) rates or symptom severity in athletes and older adults, respectively, though effects vary in impact depending on the study cohort demographics (Li et al., 2016). While these studies have not always been definitive, the decreased incidence of URTI holds potential especially when considering the possibility of defending against viruses such as COVID-19. Quecertin has been shown in one clinical study to improve innate immunity ( $\mathrm{Li}$ et al., 2016).

Another compound is anthocyanins, a subclass of bioactive phenolic compounds. Phenolics act as or influence the manifestations of antioxidants in humans, and can be found at considerable levels in several types of berries (Ayoub et al., 2016; Lee et al. 2015). While identification of anthocyanin content and types in berries has been challenging due to extraction techniques, it is known that they exhibit the antioxidant activity seen in their parent class (Ayoub et al., 2016; Lee at al., 2017). Anthocyanins in particular have been shown in mouse models to reduce inflammation by modulating biomarkers such as the aforementioned COX-2, proand anti-inflammatory cytokines, and growth factors, and affecting the movement of innate immune cells, thus raising the question of their potential activity in human patients (Peiffer et al., 2016). In humans, one study on the consumption of $34 \%$ blackcurrant anthocyanin-rich extract (BAE) by healthy adults regulated levels of circulating neutrophils and maintained the neutrophils' ability to phagocytose debris (Hurst et al., 2019). In relation to the potential antioxidant activity of anthocyanins, BAE was not found in this study to specifically affect antioxidant status, but did improve rate of recovery from oxidative stress caused by exercise, as evaluated by plasma oxidative capacity and protein carbonyl levels (Hurst et al., 2019). The research team also found that consumption of BAE prior to exercise has positive effects on innate immunity via upregulation of beneficial immune factors and anti-inflammatory activity in cells (Hurst et al., 2020). This study demonstrated that IL-10, an anti-inflammatory and antioxidant interleukin, was significantly increased in individuals who consumed BAE on a daily basis. Anthocyanins appear to assist in sustaining an optimal immune system and inflammatory balance by affecting both anti-inflammatory mediators and mediators involved in the inflammatory 
Table 1. Summary of major bioactives and metabolic compounds derived from berries that have been researched in clinical trials

\begin{tabular}{|c|c|c|}
\hline $\begin{array}{l}\text { Bioactive/meta- } \\
\text { bolic compound }\end{array}$ & Hypothesized mechanism & Potential/proven benefits \\
\hline Flavonoids & $\begin{array}{l}\text { Polyphenolic structure bestows antioxidant and anti-inflammatory } \\
\text { properties. Support gut microbiome and inhibit COX-2 }\end{array}$ & Reduce URTI rates and symptom severity \\
\hline Anthocyanins & $\begin{array}{l}\text { Modulate inflammatory biomarkers and immune response } \\
\text { cascade. Improve response to oxidative stress }\end{array}$ & $\begin{array}{l}\text { Recovery from strenuous exercise. } \\
\text { Reduce inflammation in the elderly }\end{array}$ \\
\hline Alkaloids & Modulate inflammatory biomarkers and immune response cascade & $\begin{array}{l}\text { Recovery from strenuous } \\
\text { exercise (inconclusive) }\end{array}$ \\
\hline Dietary fibers & $\begin{array}{l}\text { Increase gene expression of anti-inflammatory cytokines } \\
\text { and reduce expression of pro-inflammatory cytokines. } \\
\text { Improve antioxidant capacity and ROS processing }\end{array}$ & $\begin{array}{l}\text { Reduce inflammation and } \\
\text { support gut microbiome }\end{array}$ \\
\hline Stilbenes & $\begin{array}{l}\text { Promote anti-inflammatory chemokines and cytokines } \\
\text { to induce Th2 cells. Reduce pro-inflammatory markers in } \\
\text { plasma. Increase expression of antioxidant genes }\end{array}$ & $\begin{array}{l}\text { Treat Alzheimer's patients by improving } \\
\text { adaptive neuroimmunity. Reduce } \\
\text { inflammation in ulcerative colitis patients }\end{array}$ \\
\hline Tannins & Ellagitannins affect antioxidant capacity & $\begin{array}{l}\text { Immunoprotective via } \\
\text { antioxidant functionality }\end{array}$ \\
\hline
\end{tabular}

immune response. It is important to recognize, however, that these studies in humans are noted to be pilot studies with relatively small sample sizes of healthy adults. While encouraging, there are still limitations in attempting to extend these conclusions to individuals with medical conditions or generally poor health, or of elderly or younger age, so it is important to broaden demographic categories and sample sizes in order to draw additional conclusions. One such example is a study in older adults which demonstrated that consumption of anthocyanins in fruit juice significantly reduced serum TNF- $\alpha$, a cytokine released by immune cells which is part of natural defense, but can cause various pathological states under certain conditions (do Rosario et al., 2021). The consumption of anthocyanin-rich black chokeberry juice yielded a longitudinal reduction in the use of antibiotics to treat urinary tract infection (UTI) in elderly residents across multiple nursing homes (Handeland et al., 2014).

Alkaloids are nitrogen-containing organic compounds; some examples are caffeine (found in guarana berries) and ginsenine (found in ginseng berries) (Diel, 2020; Wang et al., 2006). Generally, clinical studies regarding berry-based alkaloids are limited. Some studies in athletes involving caffeine and ginsenine did show increased lymphocytes, NK cells, neutrophil activation, and IL10 after exercise, but the body of research is overall inconclusive (Senchina et al., 2014). While there are a considerable number of trials studying the effects of caffeine on the immune system, most of the caffeine in these studies was derived from tea or coffee fruit, as opposed to guarana.

A variety of berries are considered to be high in dietary fiber. A randomized, controlled trial involving consumption of juçara berry pulp, which is fiber-rich, by obese adults for 6 weeks increased gene expression of IL-10, but reduced the production of pro-inflammatory cytokines such as IL-6 and TNF- $\alpha$ (Santamarina et al., 2020). Another study of juçara berry as well as açaí berry, involving consumption of berry juice for 4 weeks by healthy adult subjects, improved the activity of glutathione peroxidase, which is involved in the processing of reactive oxygen species (ROS), as well as overall total antioxidant capacity (de Liz et al., 2020). The fiber of the kiwi berry has hydration and fermentation properties that may be related to its immune health effects-it improves the health of the GI microbiome (Richardson et al., 2018). As with many other phytonutrient studies, the number of berry-specific studies is limited, but results are generally promising.

Stilbenes are polyphenolic compounds that can be found in berries such as grapes and mulberries; one of the most studied stilbenes specifically derived from berries is resveratrol, which has shown encouraging beneficial effects to immunity in animal and cell models (Malaguarnera, 2019). In a relatively large sample of subjects with mild-moderate Alzheimer's disease, oral consumption of resveratrol for one year was associated with increased macrophage-derived chemokine (MDC) and IL-4 (Moussa et al., 2017). Interleukin-4 (IL-4) is involved in the anti-inflammatory response and induces both T-cell differentiation into Th2 types and alternative macrophage activation; as a synergistic effect, MDC serves to assist in the recruitment and induction of Th2 cells in the adaptive immune response against injury (Junttila, 2018). The results from the Alzheimer's study thus suggest that adaptive neuroimmunity is improved by the extended consumption of resveratrol. The administration of resveratrol to patients with ulcerative colitis over 6 weeks reduced plasma levels of C-reactive protein and TNF- $\alpha$, both of which are indicative of inflammation; in addition, antioxidant capability was improved in these patients (SamsamiKor et al., 2015). In corroboration with this study, human subjects given a diet of resveratrol with grape polyphenols showed decreased levels of pro-inflammatory cytokine genes and increased levels of antioxidant genes (Ghanim et al., 2011).

Another polyphenolic compound frequently found in berries is tannins. Myrciarcia jaboticaba, a berry-producing plant cultivated in Brazil, has beneficial antioxidant capacity by merit of its high ellagitannin content; in human subjects who consumed jaboticaba, antioxidant capacity was significantly increased (Plaza et al., 2016). Consumption of red raspberries also shows metabolism of ellagitannins, hypothetically thereby allowing them to exert their effects in immunoprotection (González-Barrio et al., 2011). Both jaboticaba and red raspberries are also notable for their anthocyanin content, which has been previously discussed in this section (Table 1).

\subsection{Specific Berries}

Blueberries are a popular variety of consumed berry that have relatively high (albeit variable) anthocyanin and phenolic acid content, 
and thus have been demonstrated to have beneficial immune-regulating effects in human cell lines and animal models (Ma et al., 2018; Correa-Betanzo et al., 2015). While the body of research for clinical trials in humans involving blueberries is smaller, there are still significant studies that have been conducted to this end. A pilot study in a small sample of healthy young women found reduction of urinary 8-hydroxydeoxyguanosine (8-OHdG) levels after consumption of blueberry juice (Kim et al., 2017). 8-OHdG is a form of reactive oxygen species (ROS)-induced lesion commonly used as a marker for DNA damage and carcinogenesis (Valavanidis et al., 2009). This study utilized vitamin C as a control for antioxidant activity and exhibited thorough controls of other factors within the study cohort. Another study in relatively healthy subjects found that daily consumption of blueberries for 6 weeks with additional consumption prior to exercise increased plasma IL10 , which was discussed in the anthocyanin section of this mini-review to have anti-inflammatory properties (McAnulty et al., 2011). This diet treatment also increased the counts of natural killer (NK) cells, which function in the innate immune system; this result may suggest a bolstering of natural immunity as a result of consistent blueberry consumption. However, small sample size and lack of 'unhealthy' subjects present as limitations to extrapolation in these two studies. Fortunately, there are some studies which address either of these issues. A well-controlled study in adult subjects with metabolic syndrome found that twice-daily consumption of blueberries significantly decreased ROS and the gene expression of cytokines frequently associated with pathological inflammation, but increased the numbers of dendritic cells, which play a significant role in adaptive immunity (Nair et al., 2017). Additionally, the research team involved in the exercise study conducted a follow-up trial in postmenopausal women and men with sedentary lifestyle habits (McAnulty et al., 2011). This study administered blueberry powder daily for 6 weeks, similar to the team's previous methodology, and also found increased counts of NK cell types; however, in contrast to several studies mentioned in this section, redox capability was not increased in the subjects of this study.

The goji berry, also known as the wolfberry, has roots in traditional Eastern medicine but has become popularized in the West for its purported health effects. Peer-reviewed research is continuing regarding the legitimate health benefits of goji berries, with a number of clinical trials involving observation of immunomodulatory activities of goji berries. A randomized, controlled trial on the consumption of a milk-based goji berry formulation by elderly human subjects, intended to observe the effects of goji berries on aging-related immune changes, found improved immunocompetence and antibody response in the elderly as measured by response to vaccination (Vidal et al., 2012). However, inflammatory markers were not significantly altered by this treatment. This study was conducted in light of previous clinical trials involving supplementation with goji berry products, but the body of research on this topic will require additional human studies to continue with significant conclusions.

Black raspberries have also been a topic of recent focus due to their potential chemopreventive effects in esophageal and colorectal cancer (Peiffer et al., 2016; Pan et al., 2015). Some of these discoveries have involved the immune-modulating effects of black raspberries and their bioactive components. Black raspberries have been shown to contain the highest extractable concentration of phenolic acids in comparison to other common berries, such as blueberries and blackberries, suggesting that their consumption leads to strong antioxidant action (Ayoub et al., 2016). In colorectal cancer patients, anthocyanins were detected in the urine after consumption of black raspberries (Wang et al., 2011). This team also found that signaling of TGF- $\beta$, a cytokine involved in immune regulation of the lymphocyte life cycle, was altered in patients with familial adenomatous polyposis, a condition which carries a high risk of colorectal cancer (Wang et al., 2014). While the precise immune system-affecting mechanisms have not yet been determined in clinical trials of black raspberries, these studies support previous research on anthocyanins and the potential for berries to treat severe malignancies in human patients.

Cranberries are frequently consumed in dried, fresh, and juice forms, with some colloquial discussion regarding the benefits of their consumption. The body of definitive clinical trials on cranberries relating to immunomodulation focuses mainly on their benefits in treating or preventing urinary tract infections (UTIs). However, many of the trials from the past decades present with poor study design. Even when study methods are better executed, results have been inconsistent. A multicenter clinical trial in adult patients with multiple sclerosis did not show significant prophylaxis of UTI when cranberry extract was consumed for one year (Gallien et al., 2014). On the other hand, the consumption of cranberry juice by a small sample of healthy adult subjects did promote the proliferation of $\gamma \delta$-T cells involved in the immune response, suggesting potential benefits in warding off influenza symptoms (Nantz et al., 2013). To address the issues of inconsistency, a study was conducted to demonstrate that whole cranberry powder, notable for its proanthocyanidin content, has significant benefits in preventing recurrent UTI in a sizeable pool of adult female subjects (Vostalova et al., 2015). This study presented a more meticulous study design and larger sample size than the research that existed previously. The team hypothesized that the greater variety of bioactive compounds found in the whole cranberry produced more definitively beneficial effects. Contemporary clinical studies on cranberries and UTI have continued to show improved study design and benefits to patients, though method of cranberry delivery is variable, with juice being most frequent (Maki et al., 2016; Foxman et al., 2015; Ledda et al., 2015; Bruyère et al., 2019). These studies frequently attribute the efficacy of cranberries as a therapy for UTI to their proanthocyanidin content. Cranberries have also shown promise in other immunoprotective effects. A clinical trial of cranberry consumption by a large study pool of adults with $\mathrm{He}$ liobacter pylori, a bacteria frequently responsible for gastrointestinal infections, showed that the consumption of cranberry juice with high levels of proanthocyanidins, but not cranberry powder, decreased rates of $H$. pylori infection and was able to strongly suppress the proliferation of the bacteria (Li et al., 2021). Clinical research into the immune effects of cranberry consumption has one of the more sizeable bodies of research in comparison to that of other berries; with a significant number of studies showing benefit across pathologies and subject demographics, modern research may benefit from seeking to determine the precise bioactives and metabolites responsible for the actions of cranberries upon the human physiology-there has been demonstration of some initiative in this field (Liu et al., 2020b, Table 2).

\section{Conclusion}

Studies on the general bioactive and metabolic compounds found in berries, or on specific berries of interest, are promising with regards to demonstrating beneficial immune system effects and reducing inflammation. It is important to note, however, that many of the studies that have been performed on berry-related immunity have been performed in animal or cell models, and that there continues to be a dearth of dedicated clinical, in vivo studies in human subjects of diverse demographics. While extrapolation from small- 
Table 2. Summary of specific berries that have been researched in clinical trials

\begin{tabular}{|c|c|c|}
\hline Berry & Hypothesized mechanism & Potential/proven benefits \\
\hline Blueberries & $\begin{array}{l}\text { Anthocyanin and phenolic acid components promote } \\
\text { antioxidant and anti-inflammatory activity }\end{array}$ & $\begin{array}{l}\text { Reduce ROS-induced lesions. Reduce exercise- } \\
\text { related inflammation. Improve adaptive immunity }\end{array}$ \\
\hline Goji berries & Unknown & $\begin{array}{l}\text { Improve immunocompetence and antibody } \\
\text { response to vaccination in the elderly }\end{array}$ \\
\hline Black raspberries & $\begin{array}{l}\text { Phenolic acids promote antioxidant } \\
\text { activity. Alter immune signaling }\end{array}$ & $\begin{array}{l}\text { Improve immune response in familial } \\
\text { adenomatous polyposis }\end{array}$ \\
\hline Cranberries & $\begin{array}{l}\text { High proanthocyanidin content. Promote beneficial } \\
\text { immune response and suppress bacterial proliferation }\end{array}$ & $\begin{array}{l}\text { Prevent or reduce recurrence of urinary tract infections. } \\
\text { Decrease rates of Heliobacter pylori infection }\end{array}$ \\
\hline
\end{tabular}

er-scale organisms to humans is certainly possible, a greater number and diversity of clinical trials will be needed before definitive conclusions can be drawn regarding the benefits of berries to the human immune system. Nonetheless, the clinical studies that have been performed have helped open the door for further research in this niche and show that berries have generally positive, or at least neutral effects on human immunity. Further studies, perhaps in consideration of general dietary practices in our modern world, will hopefully continue to elucidate the specific mechanisms of berry-modulated immune effects in the human body, and suggest therapies for patients as well as modifications that the general public can make to better guard against malignant conditions.

\section{References}

Ayoub, M., de Camargo, A.C., and Shahidi, F. (2016). Antioxidants and bioactives of free, esterified and insoluble-bound phenolics from berry seed meals. Food Chem. 197: 221-232.

Brown, K., DeCoffe, D., Molcan, E., and Gibson, D.L. (2012). Diet-induced dysbiosis of the intestinal microbiota and the effects on immunity and disease. Nutrients 4(8): 1095-1119.

Bruyère, F., Azzouzi, A.R., Lavigne, J.P., Droupy, S., Coloby, P., Game, X., Karsenty, G., Issartel, B., Ruffion, A., Misrai, V., Sotto, A., and Allaert, F.A. (2019). A multicenter, randomized, placebo-controlled study evaluating the efficacy of a combination of propolis and cranberry (Vaccinium macrocarpon) (DUAB ${ }^{\circledR}$ ) in preventing low urinary tract infection recurrence in women complaining of recurrent cystitis. Urol. Int. 103(1): 41-48.

Correa-Betanzo, J., Padmanabhan, P., Corredig, M., Subramanian, J., and Paliyath, G. (2015). Complex formation of blueberry (Vaccinium angustifolium) anthocyanins during freeze-drying and its influence on their biology activity. J. Agric. Food Chem. 63(11): 2935-2946.

de Liz, S., Cardoso, A.L., Copetti, C.L.K., de Fragas Hinnig, P., Vieira, F.G.K., da Silva, E.L., Schulz, M., Fett, R., Micke, G.A., and Di Pietro, P.F. (2020). Açaí (Euterpe oleracea Mart.) and juçara (Euterpe edulis Mart.) juices improved HDL-c levels and antioxidant defense of healthy adults in a 4-week randomized cross-over study. Clin. Nutr. 39(12): 3629-3636.

Diel, P. (2020). Caffeine and doping-what we have learned since 2004. Nutrients 12: 2167.

Do Rosario, V.A., Fitzgerald, Z., Broyd, S., Paterson, A., Roodenrys, S., Thomas, S., Bliokas, V., Potter, J., Walton, K., Weston-Green, K., Yousefi, M., Williams, D., Wright, I.M.R., and Charlton, K. (2021). Food anthocyanins decrease concentrations of TNF- $\alpha$ in older adults with mild cognitive impairment: a randomized, controlled, double blind clinical trial. Nutr., Metab. Cardiovasc. Dis. 31(3): 950-960.

Foxman, B., Cronenwett, A.E.W., Spino, C., Berger, M.B., and Morgan, D.M. (2015). Cranberry juice capsules and urinary tract infection after surgery: results of a randomized trial. Am. J. Obstet. Gynecol. 213(2): 194.e1-8.

Gallien, P., Amarenco, G., Benoit, N., Bonniaud, V., Donzé, C., Kerdraon, J., de Seze, M., Denys, P., Renault, A., Naudet, F., and Reymann, J.M.
(2014). Cranberry versus placebo in the prevention of urinary infections in multiple sclerosis: a multicenter, randomized, placebo-controlled, double-blind trial. Mult. Scler. 20(9): 1252-1259.

Ghanim, H., Sia, C.L., Korzeniewski, K., Lohano, T., Abuaysheh, S., Marumganti, A., Chaudhuri, A., and Dandona, P. (2011). A resveratrol and polyphenol preparation suppresses oxidative and inflammatory stress response to a high-fat, high-carbohydrate meal. J. Clin. Endocrinol. Metab. 96(5): 1409-1414.

González-Barrio, R., Edwards, C.A., and Crozier, A. (2011). Colonic catabolism of ellagitannins, ellagic acid, and raspberry anthocyanins: in vivo and in vitro studies. Drug Metab. Dispos. 39(9): 1680-1688.

Gupta, C., and Prakash, D. (2014). Phytonutrients as therapeutic agents. J. Complement Integr. Med. 11(3): 151-169.

Handeland, M., Grude, N., Torp, T., and Slimestad, R. (2014). Black chokeberry juice (Aronia melanocarpa) reduces incidences of urinary tract infection among nursing home residents in the long term--a pilot study. Nutr Res. 34(6): 518-525.

Hooper, L.V. (2011). You AhR what you eat: linking diet and immunity. Cell 147(3): 489-491.

Hurst, R.D., Lyall, K.A., Roberts, J.M., Perthaner, A., Wells, R.W., Cooney, J.M., Jensen, D.J., Burr, N.S., and Hurst, S.M. (2019). Consumption of an anthocyanin-rich extract made from New Zealand blackcurrants prior to exercise may assist recovery from oxidative stress and maintains circulating neutrophil function: a pilot study. Front. Nutr. 6: 73 .

Hurst, R.D., Lyall, K.A., Wells, R.W., Sawyer, G.M., Lomiwes, D., Ngametua, N., and Hurst, S.M. (2020). Daily consumption of an anthocyanin-rich extract made from New Zealand blackcurrants for 5 weeks supports exercise recovery through the management of oxidative stress and inflammation: a randomized placebo controlled pilot study. Front. Nutr. 7: 16

Junttila, I.S. (2018). Tuning the cytokine responses: an update on interleukin (IL)-4 and IL-13 receptor complexes. Front. Immunol. 9: 888

Kim, M., Na, H., Kasai, H., Kawai, K., Li, Y.S., and Yang, M. (2017). Comparison of blueberry (Vaccinium spp.) and vitamin C via antioxidative and epigenetic effects in human. J. Cancer Prev. 22(3): 174-181.

Ledda, A., Bottari, A., Luzzi, R., Belcaro, G., Hu, S., Dugall, M., Hosoi, M., Ippolito, E., Corsi, M., Gizzi, G., Morazzoni, P., Riva, A., Giacomelli, L., and Togni, S. (2015). Cranberry supplementation in the prevention of non-severe lower urinary tract infections: a pilot study. Eur. Rev. Med. Pharmacol. Sci. 19(1): 77-80.

Lee, S.G., Vance, T.M., Nam, T.G., Kim, D.O., Koo, S.I., and Chun, O.K. (2015). Contribution of anthocyanin composition to total antioxidant capacity of berries. Plant Foods Hum. Nutr. 70(4): 427-32.

Lee, Y.M., Yoon, Y., Yoon, H., Park, H.M., Song, S., and Yeum, K.J. (2017). Dietary anthocyanins against obesity and inflammation. Nutrients 9(10): 1089.

Li, Y., Yao, J., Han, C., Yang, J., Chaudhry, M.T., Wang, S., Liu, H., and Yin, Y. (2016). Quecertin, inflammation and immunity. Nutrients $8(3): 167$.

Li, Z.X., Ma, J.L., Guo, Y., Liu, W.D., Li, M., Zhang, L.F., Zhang, Y., Zhou, T., Zhang, J.Y., Gao, H.E., Guo, X.Y., Ye, D.M., Li, W.Q., You, W.C., and Pan, K.F. (2021). Suppression of Helicobacter pylori infection by daily cranberry intake: a double-blind, randomized, placebo-controlled trial. J. Gastroenterol. Hepatol. 36(4): 927-935.

Liu, H., Garrett, T.J., Su, Z., Khoo, C., Zhao, S., and Gu, L. (2020a). Modifications of the urinary metabolome in young women after cranberry 
juice consumption were revealed using the UHPLC-Q-orbitrap-HRMSbased metabolomics approach. Food Funct. 11(3): 2466-2476.

Liu, M., Gao, Y., Zhang, Y., Shuzhen, S., Chen, Y., and Tian, J. (2020b). The association between severe or dead COVID-19 and autoimmune diseases: a systematic review and meta-analysis. J. Infect. 81(3): E93E95.

Ma, L., Sun, Z., Zeng, Y., Luo, M., and Yang, J. (2018). Molecular mechanism and health role of functional ingredients in blueberry for chronic disease in human beings. Int. J. Mol. Sci. 19(9): 2785.

Maki, K.C., Kaspar, K.L., Khoo, C., Derrig, L.H., Schild, A.L., and Gupta, K. (2016). Consumption of a cranberry juice beverage lowered the number of clinical urinary tract infection episodes in women with a recent history of urinary tract infection. Am. J. Clin. Nutr. 103(6): 1434-1442.

Malaguarnera, L. (2019). Influence of resveratrol on the immune response. Nutrients 11(5): 946 .

McAnulty, L.S., Nieman, D.C., Dumke, C.L., Shooter, L.A., Henson, D.A., Utter, A.C., Milne, G., and McAnulty, S.R. (2011). Effect of blueberry ingestion on natural killer cell counts, oxidative stress, and inflammation prior to and after $2.5 \mathrm{~h}$ of running. Appl. Physiol. Nutr. Metab. 36(6): 976-984.

Moussa, C., Hebron, M., Huang, X., Ahn, J., Rissman, R.A., Aisen, P.S., and Turner, R.S. (2017). Resveratrol regulates neuro-inflammation and induces adaptive immunity in Alzheimer's disease. J. Neuroinflammation 14(1): 1.

Myles, I.A. (2014). Fast food fever: reviewing the impacts of the Western diet on immunity. Nutr. J. 13: 61.

Nair, A.R., Mariappan, N., Stull, A.J., and Francis, J. (2017). Blueberry supplementation attenuates oxidative stress within monocytes and modulates immune cell levels in adults with metabolic syndrome: a randomized, double-blind, placebo-controlled trial. Food Func. 8(11): 4118-4128.

Nantz, M.P., Rowe, C.A., Muller, C., Creasy, R., Colee, J., Khoo, C., and Percival, S.S. (2013). Consumption of cranberry polyphenols enhances human $\gamma \delta-T$ cell proliferation and reduces the number of symptoms associated with colds and influenza: a randomized, placebo-controlled intervention study. Nutr. J. 12: 161.

Pan, P., Skaer, C.W., Stirdivant, S.M., Young, M.R., Stoner, G.D., Lechner, J.F., Huang, Y.W., and Wang, L.S. (2015). Beneficial regulation of metabolic profiles by black raspberries in human colorectal cancer patients. Cancer Prev. Res. (Phila) 8(8): 743-750.

Peiffer, D.S., Wang, L.S., Zimmerman, N.P., Ransom, B.W.S., Carmella, S.G., Kuo, C.T., Chen, J.H., Oshima, K., Huang, Y.W., Hecht, S.S., and Stoner, G.D. (2016). Dietary consumption of black raspberries or their anthocyanin constituents alters innate immune cell trafficking in esophageal cancer. Cancer Immuno. Res. 4(1): 72-82.

Pérez-Cano, F.J., and Castell, M. (2016). Flavonoids, inflammation and immune system. Nutrients 8(10): 659.

Plaza, M., Batista, Â.G., Cazarin, C.B.B., Sandahl, M., Turner, C., Östman, E., and Júnior, M.R.M. (2016). Characterization of antioxidant polyphenols from Myrciaria jaboticaba peel and their effects on glucose metabolism and antioxidant status: a pilot clinical study. Food Chem. 211: 185-197.

Richardson, D.P., Ansell, J., and Drummond, L.N. (2018). The nutritional and health attributes of kiwifruit: a review. Eur. J. Nutr. 57)8): 26592676.

Ricordi, C., Garcia-Contreras, M., and Farnetti, S. (2015). Diet and inflammation: possible effects on immunity, chronic diseases, and life span. J. Am. Coll. Nutr. 34(1): 10-13.

Samsami-Kor, M., Daryani, N.E., Asl, P.R., and Hekmatdoost, A. (2015). Anti-inflammatory effects of resveratrol in patients with ulcerative colitis: a randomized, double-blind, placebo-controlled pilot study. Arch. Med. Res. 46(4): 280-285.

Santamarina, A.B., Jamar, G., Mennitti, L.V., de Cássia Cesar, H., Vasconcelos, J.R., Oyama, L.M., de Rosso, V.V., and Pisani, L.P. (2020). Obesity-related inflammatory modulation by juçara berry (Euterpe edulis Mart.) supplementation in Brazilian adults: a double-blind randomized controlled trial. Eur. J. Nutr. 59(4): 1693-1705.

Senchina, D.S., Hallam, J.E., Kohut, M.L., Nguyen, N.A., d, N., and Perera, M.A. (2014). Alkaloids and athlete immune function: caffeine, theophylline, gingerol, ephedrine, and their congeners. Exerc. Immunol. Rev. 20: 68-93.

Tilg, H., and Moschen, A.R. (2015). Food, immunity, and the microbiome. Gastroenterology 148(6): 1107-1119.

Valavanidis, A., Vlachogianni, T., and Fiotakis, C. (2009). 8-hydroxy-2'deoxyguanosine (8-OHdG): a critical biomarker of oxidative stress and carcinogenesis. J. Environ. Sci. Health C Environ. Carcinog. Ecotoxicol. Rev. 27(2): 120-139.

Vezza, T., Rodriguez-Nogales, A., Algieri, F., Utrilla, M.P., Rodriguez-Cabezas, M.E., and Galvez, J. (2016). Flavonoids in inflammatory bowel disease: a review. Nutrients 8(4): 211.

Vidal, K., Bucheli, P., Gao, Q., Moulin, J., Shen, L.S., Wang, J., Blum, S., and Benyacoub, J. (2012). Immunomodulatory effects of dietary supplementation with a milk-based wolfberry formulation in healthy elderly: a randomized, double-blind, placebo-controlled trial. Rejuvenation Res. 15(1): 89-97.

Vostalova, J., Vidlar, A., Simanek, V., Galandakova, A., Kosina, P., Vacek, J., Vrbkova, J., Zimmermann, B.F., Ulrichova, J., and Student, V. (2015) Are high proanthocyanidins key to cranberry efficacy in the prevention of recurrent urinary tract infection? Phytother. Res. 29(10): 1559-1567.

Wang, J.Y., Li, X.G., and Yang, X.W. (2006). Ginsenine, a new alkaloid from the berry of Panax ginseng C. A. Meyer. J. Asian Nat. Prod. Res. 8(7): 605-608.

Wang, L.S., Arnold, M., Huang, Y.W., Sardo, C., Seguin, C., Martin, E., Huang, T.H.M., Riedl, K., Schwartz, S., Frankel, W., Pearl, D., Xu, Y., Winston, J., Yang, G.Y., and Stoner, G. (2011). Modulation of genetic and epigenetic biomarkers of colorectal cancer in humans by black raspberries: a phase I pilot study. Clin. Cancer Res. 17(3): 598-610.

Wang, L.S., Burke, C.A., Hasson, H., Kuo, C.T., Sardo Molmenti, C.L., Seguin, C., Liu, P., Huang, T.M., Frankel, W.L., and Stoner, G.D. (2014). A Phase Ib study of the effects of black raspberries on rectal polyps in patients with familial adenomatous polyposis. Cancer Prev. Res. 7(7): 666-674.

Wypych, T.P., Marsland, B.J., and Ubags, N.D.J. (2017). The impact of diet on immunity and respiratory diseases. Annals ATS. 14(5): S339-S347.

Yazdanpanah, F., Hamblin, M.R., and Rezaei, N. (2020). The immune system and COVID-19: friend or foe? Life Sci. 256: 117900. 